\title{
BMJ Open Quality Introducing an electronic tracking tool into daily multidisciplinary discharge rounds on a medicine service: a quality improvement project to reduce length of stay
}

Nicholas Meo, Evan Paul, Christopher Wilson, Janice Powers, Marinette Magbual, Kari Mae Miles

To cite: Meo N, Paul E, Wilson C, et al. Introducing an electronic tracking tool into daily multidisciplinary discharge rounds on a medicine service: a quality improvement project to reduce length of stay.BMJ Open Quality 2018;7:e000174. doi:10.1136/ bmjoq-2017-000174

Received 31 July 2017 Revised 31 May 2018 Accepted 14 June 2018
Check for updates

(C) Author(s) (or their employer(s)) 2018. Re-use permitted under CC BY-NC. No commercial re-use. See rights and permissions. Published by BMJ.

Hospital and Speciality Medicine, VA Puget Sound Health Care System, Seattle, Washington, USA

Correspondence to Dr Nicholas Meo; nicholas.meo@va.gov

\section{ABSTRACT}

Background Inefficient coordination of care around discharge can increase length of stay, lead to ineffective transitions and contribute an unnecessary cost burden to patients and hospital systems. Multidisciplinary discharge rounds can improve situational awareness among team members leading to more efficient and better coordinated care. This project aimed to standardise the daily discharge rounds occurring on a medicine service to reduce length of stay. Participants included physicians, nurses and social workers.

Methods A key driver diagram was developed to understand drivers of length of stay. Improving multidisciplinary care coordination was targeted as an initial area of focus. Stakeholder interviews were held to understand current participants challenges with the daily discharge rounds process. Baseline assessment included a review of discharges for 6 weeks before the initial intervention. A Plan Do Study Act quality improvement framework was used to implement change.

Intervention An electronic tool was developed which highlighted critical information to be captured during discharge rounds on each current inpatient in a standardised fashion. Information was reviewed and solicited from care teams by a facilitator, then edited and displayed in real time to all team members by a scribe. Results The average length of stay decreased by 1.4 days $(p<0.05)$, an improvement of $21.1 \%$. There was no measured increase on readmission rate during the intervention period.

Conclusion An electronic tool to standardise information gathered among team members in daily discharge rounds led to improvements in length of stay. Multidisciplinary discharge rounds are an important venue for discharge planning across inpatient care teams and efforts to optimise communication between team members can improve care.

\section{INTRODUCTION}

Length of stay is an important benchmark for inpatient care both in terms of care delivery and administrative oversight. Multiple studies have shown that an increased length of stay is associated with worse outcomes in a variety of acute medical scenarios. ${ }^{12}$ One factor that prolongs length of stay is inefficient coordination of care around discharge, which leads to ineffective transitions and contributes unnecessary cost burden to patients and hospital systems. Interventions to improve discharge planning can lead to demonstrated decreases in length of stay, reductions in readmission rates and improvements in satisfaction among patients and providers. ${ }^{34}$

Multidisciplinary discharge rounds can improve situational awareness among team members leading to more efficient and better coordinated care. These meetings often occur daily among physicians, nurses, social workers and other health professionals caring for a cohort of patients, but best practices around communication within these teams are not well described. A variety of communication tools have been used to improve communication within multidisciplinary teams. The use of whiteboards for multidisciplinary information sharing has been studied, but a physical system such as this creates potential issues regarding inconsistent use, staff buy-in and patient privacy. ${ }^{56}$ Several institutions have instead used the EHR to improve care team communication leading to improvements in early discharge, ${ }^{7-10}$ and emergency department flow ${ }^{11}$ however, not within the setting of discharge rounds.

The length of stay on our local medicine service had been increasing over time on hospital-based performance measures, impacting inpatient flow throughout the facility and increasing the number of patients boarding in the emergency department. A task force was formed comprising members from nursing, hospitalist medicine, social work services and analytics to identify opportunities to address length of stay. Leveraging 
the collective expertise of a multidisciplinary team and using improvement methodology, we aimed to reduce average length of stay at our facility, with a goal of $10 \%$ reduction within 2 months. A secondary goal of the project was to improve care team satisfaction and morale.

\section{METHODS}

Setting

This project took place at a 100 medical-surgical bed Veteran Affairs hospital in an urban, academic setting. The medicine service comprised four internal medicine resident led teams, two hospitalist led teams, one cardiology team and one neurology team. Resident teams on the medicine service comprised an internal medicine attending physician, a PGY2 (postgraduate) senior internal medicine resident, two PGY1 interns from internal medicine, anaesthesia, psychiatry or family medicine residencies and 1-2 medical students. The cardiology team comprised a cardiology attending and four PGY2 internal medicine residents and the neurology team comprised a neurology attending and four neurology PGY2s. Admissions averaged 64 per week across all teams.

At baseline, multidisciplinary discharge rounds took place each non-holiday weekday from 08:30 hours to 09:50 hours with social workers, administrative assistants and charge nurses from each unit. Utilisation management reviewers, a home health coordinator and the admissions coordinator from the Community Living
Centre (a local Veteran Affairs skilled nursing facility) joined via a concurrent telephone conference. Each medical team rotated through the huddle in $10 \mathrm{~min}$ intervals and discussed the discharge needs of each patient on their census. The conversation was initiated and the topics discussed were led by the physician (medical student, resident or attending depending on team preference). There was an average of 64 admissions per week to the medicine services during the baseline and intervention periods, and teams had no more than 18 patients discussed during the rounds as this was the maximal limit to an individual team census per hospital policy.

\section{Design}

A task force comprising local champions from hospital medicine, nursing, social work and information technology met weekly to discuss improvements that could be made to improve patient flow. A key driver diagram was constructed to identify possible intervention points (figure 1). The improvement effort was scoped to focus on the daily multidisciplinary discharge rounding process and the task force set a specific performance aim. Baseline stakeholder interviews were held with members of discharge rounds to identify current challenges with the process. Results of interviews were discussed within the task force and themes informed the design of initial interventions. A Plan Do Study Act (PDSA) improvement framework was used to implement change.

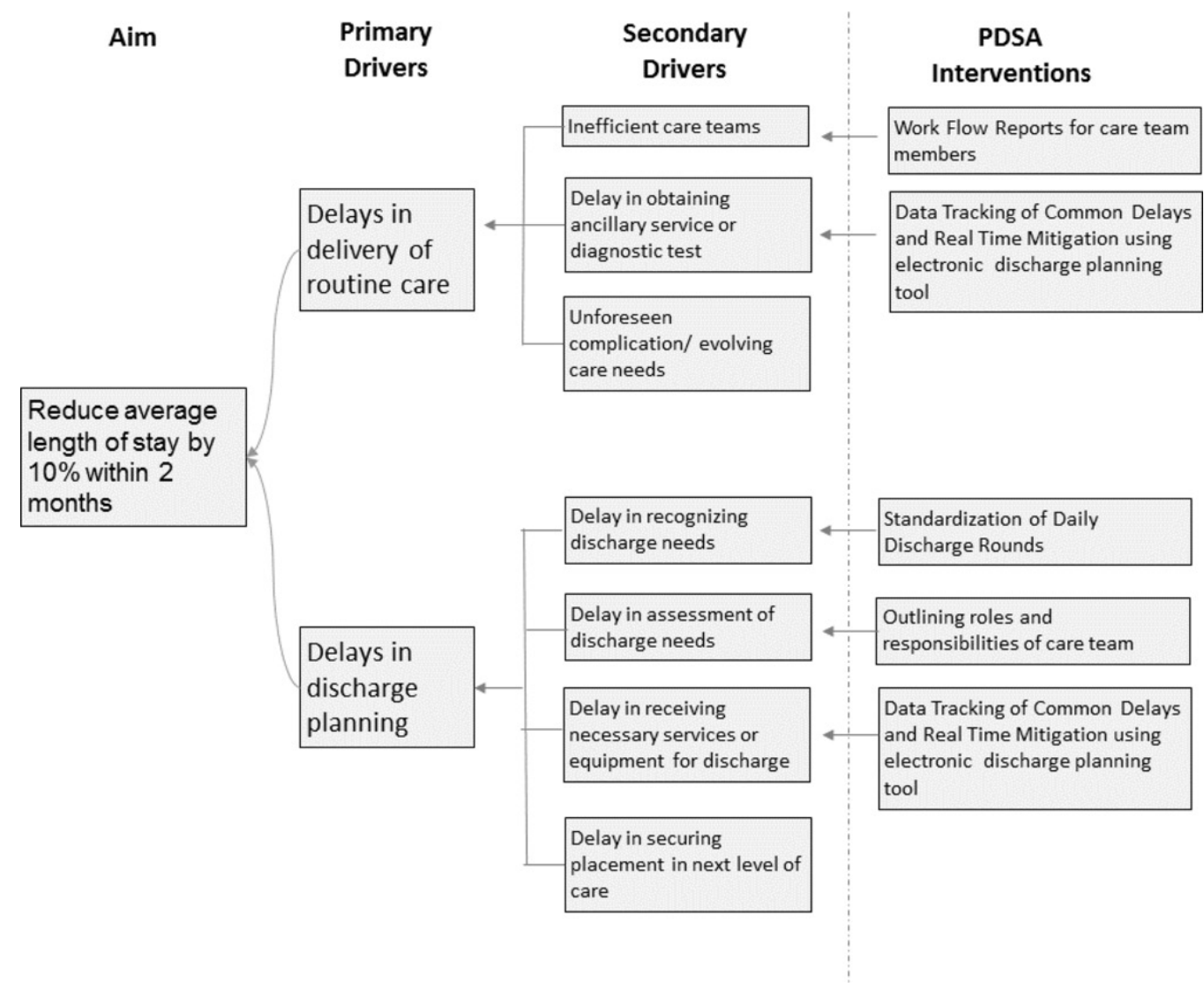

Figure 1 A key driver diagram. 


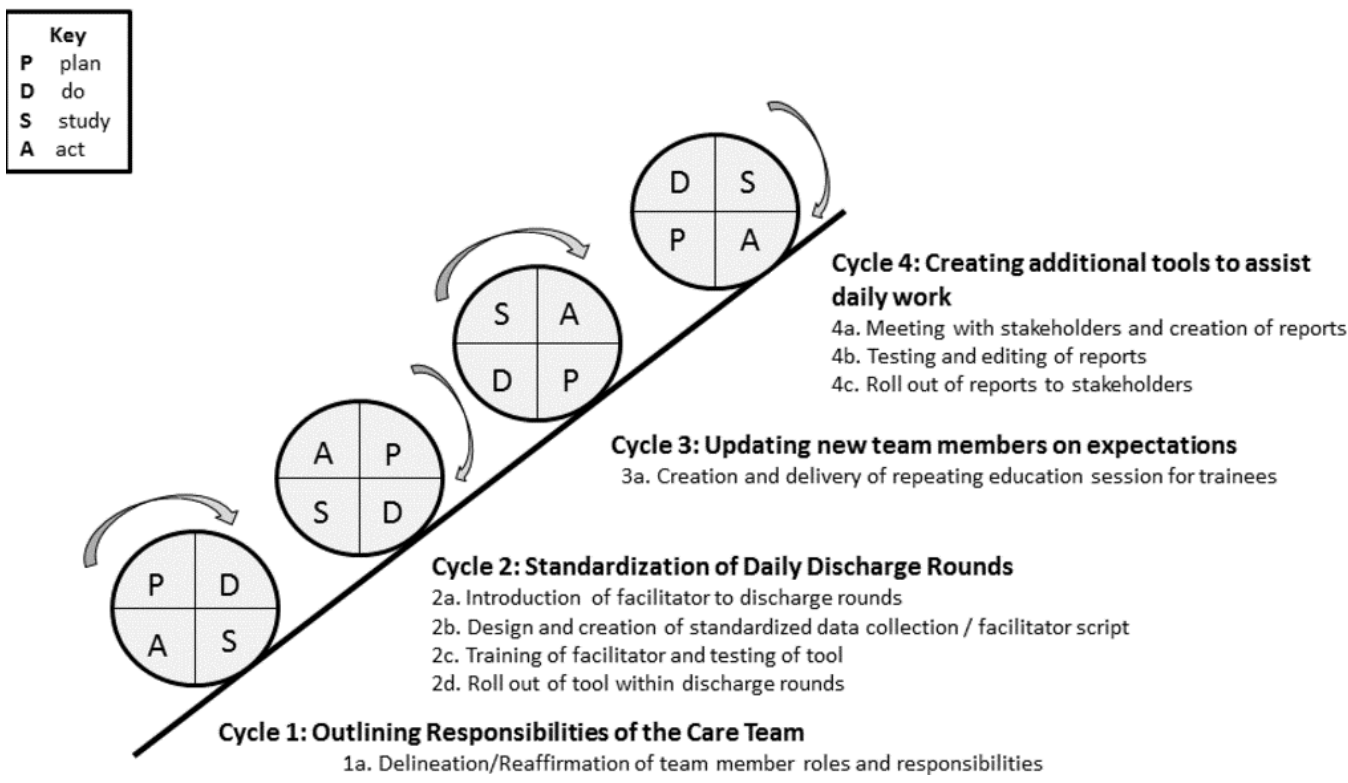

Figure 2 PDSA testing ramp for improvements to discharge rounds.

\section{Strategy}

Iterative tests of change using PDSA cycle methodology continuously adapted future work. Here we describe the successive PDSA cycles over the intervention period (figure 2).

\section{Outlining responsibilities of the care team}

The roles and responsibilities of each team member, ground rules centred on mutual respect and goals for communication during discharge rounds were articulated to all and posted in the meeting rooms. The role of a facilitator was then introduced to the discharge rounds process to guide conversations. The facilitator was a hospitalist not currently on service, the manager of acute care social work or the nurse manager of the patient flow department. Regardless of position, the facilitator used a standard script developed by the task force to capture key elements of the discharge planning process.

\section{Standardisation of daily discharge rounds}

An electronic discharge planning tool was deployed to both display and capture key information during daily discharge rounds. It displayed the patient's name, medical record number, patient location, team, attending name, admission diagnosis, admission date, current length of stay, upcoming appointments and primary care provider. This information was pulled automatically from the medical record on each patient. The tool prompted key information to be collected from care teams including estimated day of discharge, estimated disposition, anticipated discharge needs, date when medically ready for discharge, delays in care noted by care teams and barriers to care. A status field was added to collect additional useful information discussed during the huddle (figure 3). The tool was displayed on a large screen monitor in real time during the huddle. This information contained in the tool matched the script used by the facilitator. The discussion from all members of the care team was summarised and entered into the tool by a scribe.

The data tool itself was created as an intermediate database that combined information from a variety of different data resources as well as information captured during the morning discharge rounds. Administrative data were retrieved from the Veteran Health Administration's data warehouse through a series of SQL queries, which was then associated with data from our live bed management system and pushed through to a user interface developed in Microsoft Access. This allowed for users to have a user-friendly tool that helped guide discharge rounds. Information was stored on each patient in a registry and allowed for novel reporting capabilities as well as tracking of aggregate results over time.

\section{Updating new team members on expectations}

Several weeks into the intervention period, it was noted during weekly improvement team meetings that the resident led teams completing rotations at the hospital could be better orientated to the changes that occurred in daily discharge rounds and a 10 min educational intervention was created and subsequently administered each month to the incoming group of rotating trainees.

\section{Creating additional tools to assist daily work}

To extend the successes from previous cycles of improvement, the task force wanted to further leverage the information stored within the huddle tool. Team members were queried about aggregated data that would assist with their daily work efficiency. Multiple distinct summary reports from the tool were then generated for a variety of care coordination purposes. These reports were automated and could be run by any care team provider and displayed information collected within the electronic discharge planning tool. A patient census report displayed all patients by team or ward and the most 


\section{Remove Team \\ Filter By Selected Team \\ Search by

\section{Patient}

Sex

Bed

Treating Specialty

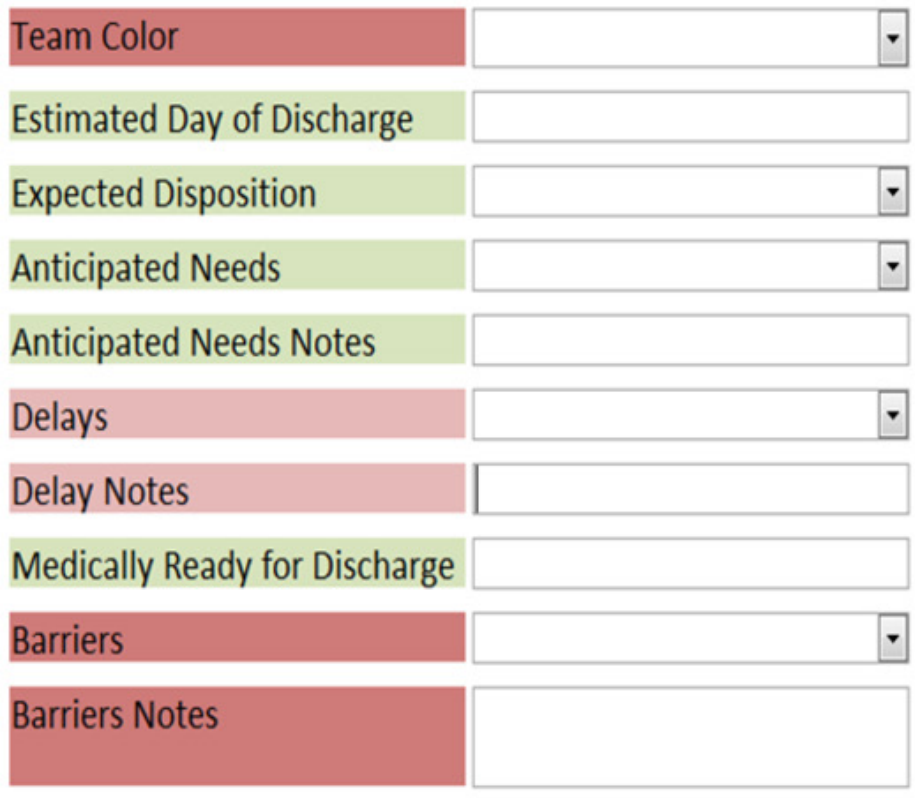

\section{Status}

Figure 3 User interface for an electronic discharge planning tool
Admit Diagnosis

Attending Physician

Treating Specialty

Length of Stay

Admission Date

Current Ward

Service Connection \%

Primary Care Provider

\section{Temp}

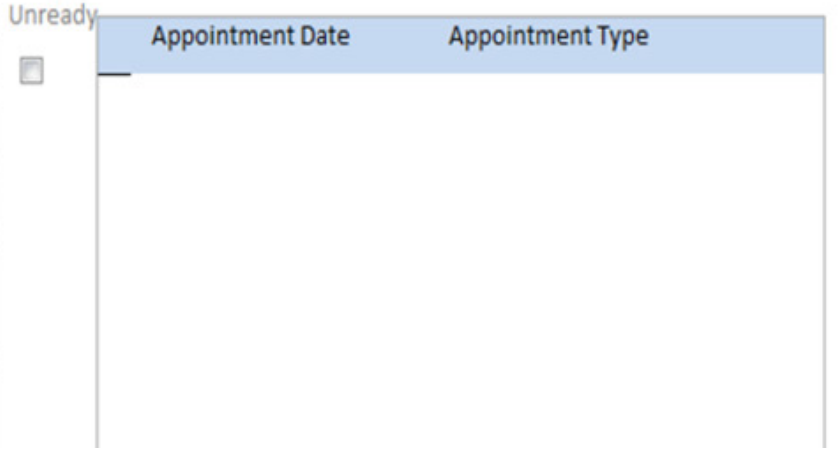

updated information contained within the status field of the electronic tool. A postdischarge rounds action list report displayed patients anticipated to discharge in the next 48 hours and itemised tasks needing to be completed in the interim period. A report of all medically ready for discharge patients and their current barriers to discharge was generated for hospital leadership and the admissions coordinator at the Community Living Center. These data tools were developed simultaneously and made available to any care provider.

\section{Measurement}

Unadjusted length of stay was measured on each discharged medicine, neurology and cardiology patient and an average was calculated. Results were determined weekly, by admission date. The average length of stay was selected as the primary outcome measure. However, very extended length of stays ( $>90$ days) due to complex patient funding, guardianship or disposition needs were excluded from calculations. Median length of stay was also determined. Thirty-day readmission rates in the precohorts and postcohorts were compared as a balancing measure. The outcome measure was plotted on a control chart over time. Two sample t-tests were used to compare means in the preintervention and postintervention phases of the project.

Minitab, V.18 was used for data analysis. The local research ethics committee deemed this project not human subjects research and the facility quality department approved this improvement initiative.

\section{RESULTS}

The introduction of an electronic tool, in conjunction with team member role definition and a facilitated huddle using a script led to a decrease in the average length of stay of 1.4 days $(\mathrm{p}<0.05)$. This was a $21.1 \%$ reduction from the baseline level (figure 4 ). The range of the weekly median length of stay in the preintervention phase was 3.0-4.5 days and in the postintervention phase was 2.0-3.0 days.

Delays in discharge coordination were identified as a primary driver target for future improvement efforts and improving the existing multidisciplinary discharge rounds became the focus of the project. Stakeholder interviews were conducted with 13 staff, including 


\section{Xbar Chart of Averge Length of Stay}

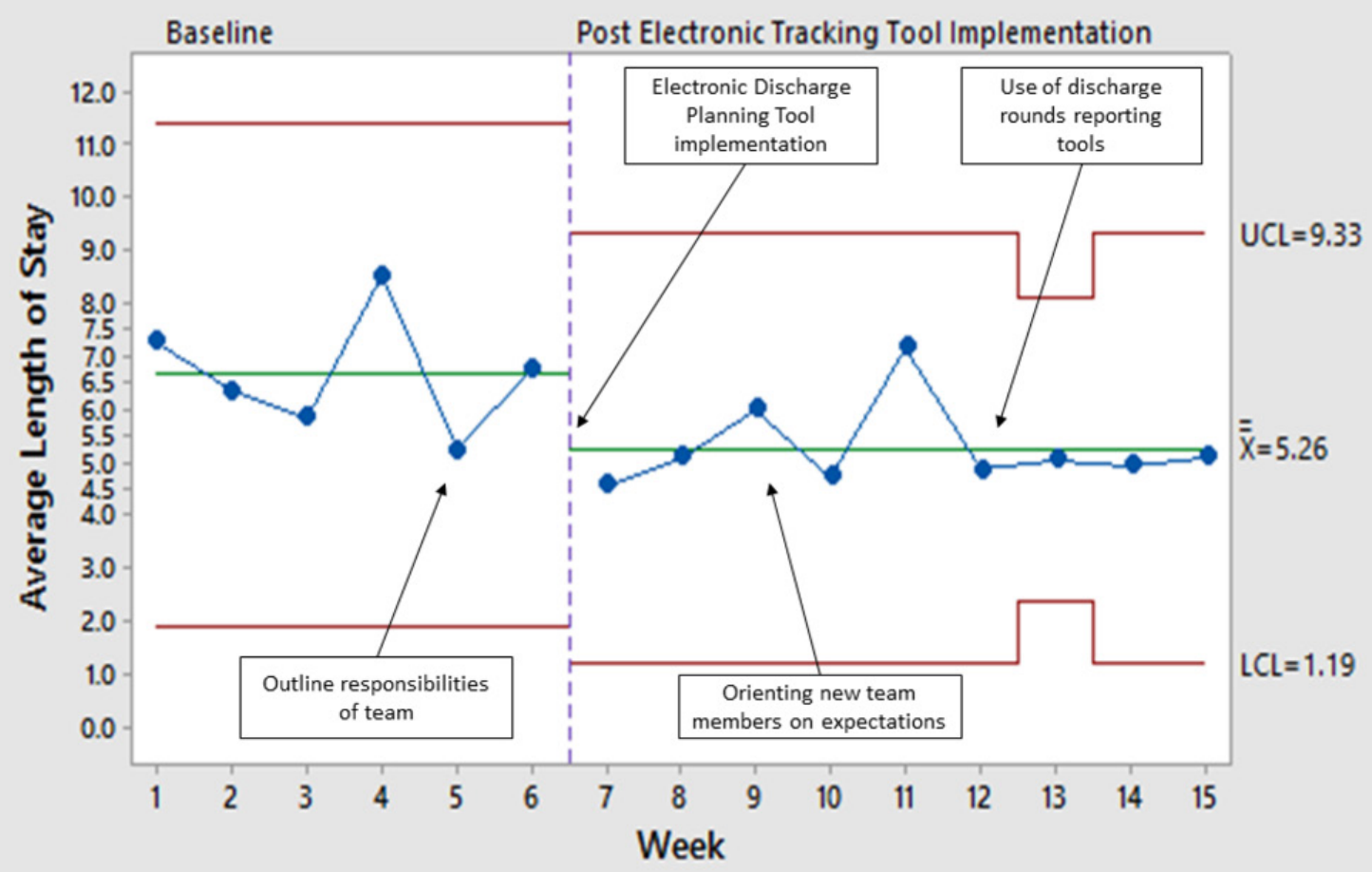

Figure 4 Control chart of average length of stay. LCL, lower control limit; UCL, upper control limit.

nurses, physicians and social workers who participated in discharge rounds regularly. Forty-six per cent reported being only 'somewhat satisfied' and no participants rated their current satisfaction with discharge rounds as 'completely satisfied'. Challenges identified included role delineation, variability of the usefulness of discussion during rounds to complete tasks for the day and variability within care team's ability to readily identify discharge needs.

The morning huddle took place with use of the tool on every non-holiday weekday except for 6 days due to computer malfunctions which limited access to the electronic tool. On several occasions, one of the seven physician teams did not arrive on time to participate due to competing clinical care demands. Although not explicitly tracked, this was estimated to occur on less than five occasions. Each current inpatient was discussed in discharge rounds during the baseline and intervention period. At no point did the discharge rounds run past 10:00 hours in the baseline or intervention period.

The initial control chart demonstrated special cause variation in the postintervention period, 4 out of 5 points were more than $1 \mathrm{SD}$ from the centre line. ${ }^{12} \mathrm{~A}$ new centre line was therefore determined for the postintervention period. Before implementation of the electronic tool, the 30 -day readmission rate was $15.68 \%$ and afterwards $15.79 \%$. Participants in discharge rounds found the tool useful to streamline and standardise communication.

\section{DISCUSSION}

Over a 10-week period, there was a reduction in the length of stay after implementation of an electronic tracking tool to standardise discharge rounds. The changes to discharge rounds did not prolong the length of the process significantly and appeared to be well received by staff participants. There was no change in the readmission rate during the observation period.

Standardising communication across multidisciplinary teams was a strength of this intervention and was assisted by a process-embedded electronic tool. This quality improvement initiative adds to a growing base of process oriented information technology tools to assist multidisciplinary rounds. ${ }^{781113}$ It also further reinforces the benefit of discharge process standardisation to decrease length of stay. ${ }^{410}$ Collecting information from discharge rounds in real time and digitally also allowed for the creation of aggregated reports to assist with a variety of staff member daily workflows. This heightened the perceived value of information discussed during discharge rounds for participants.

A novel aspect of this improvement initiative was the previously undescribed role of a facilitator within multidisciplinary discharge rounds. The facilitator led the meeting using a standardised script to ensure critical information was captured and in doing so was able make sure each care team members voice was heard. This contributed to improvements in communication and over the weeks of the intervention promoted a positive 
team culture with a shared vision of proactive discharge planning. The implementation of a structured daily multidisciplinary discharge rounds paired with an information technology tool for real time data capture may be generalisable to other acute care settings and may be particularly effective in settings with trainees.

\section{Lessons and limitations}

This process was created using the input of front-line users, who were motivated by the prospect of improvements in their daily workflow. The significant support buy-in from executive leadership also benefitted the project greatly. Importantly, the efficacy of this project was highly dependent on frequent and rigorous maintenance in the form of weekly meetings to maintain momentum and adapt to unforeseen barriers are they arose. There are several limitations of this study, including improvements in the length of stay could be in part due to patient characteristics or provider changes that occurred during the intervention period; however, the same pool of attending physicians rotated on service during the entire study period. Discharge rounds were not conducted on weekends and holidays due to the staffing of facilitators. However, it is our hope to extend the discharge rounds into the weekends in the future. There were also a limited number of occasions where the electronic tool was not able to be used during the intervention period due to unforeseen technological complications or competing clinical care demands, though these occasions were rare.

\section{Conclusion}

Our findings suggest that standardisation of communication during discharge rounds can have a significant impact on length of stay. We surpassed our initial targeted goal within our aim statement. The intervention felt to be the most impactful was the electronic tracking tool embedded into existing process workflow. This was a sustainable intervention that will ideally continue to assist in team communication into the future. Going forward, our hope is that the regular collection of delays and barriers from discharge rounds will allow for further targeted process improvement. We believe tools such as this, which standardised communication and data collection related to discharge, could prove useful to a variety of inpatient facilities in the future.
Acknowledgements Marcus Grandjean, Pamela Popplewell, Connie Morantes, Reuben Argel, Bethany Stumpf, Jessica Holman.

Contributors NM contributed to study design, monitored data collection, performed statistical analysis, analysed data, drafted and revised the paper. EP analysed data and revised the paper. CW contributed to study design, designed data tracking tools, monitored data collection, performed statistical analysis, analysed data and revised the paper. JP, MM and KM contributed to study design, analysed data and revised the paper.

Funding The authors have not declared a specific grant for this research from any funding agency in the public, commercial or not-for-profit sectors.

Competing interests None declared.

Provenance and peer review Not commissioned; externally peer reviewed.

Open access This is an open access article distributed in accordance with the Creative Commons Attribution Non Commercial (CC BY-NC 4.0) license, which permits others to distribute, remix, adapt, build upon this work non-commercially, and license their derivative works on different terms, provided the original work is properly cited, appropriate credit is given, any changes made indicated, and the use is non-commercial. See: http://creativecommons.org/licenses/by-nc/4.0/.

\section{REFERENCES}

1. Rachoin JS, Skaf J, Cerceo E, et al. The impact of hospitalists on length of stay and costs: systematic review and meta-analysis. Am J Manag Care 2012;18:e23-e30.

2. Carek PJ, Boggan H, Mainous AG, et al. Inpatient care in a community hospital: comparing length of stay and costs among teaching, hospitalist, and community services. Fam Med 2008;40:119-24.

3. Gonçalves-Bradley DC, Lannin NA, Clemson LM, et al. Discharge planning from hospital. Cochrane Database Syst Rev 2016;23.

4. Ortiga B, Salazar A, Jovell A, et al. Standardizing admission and discharge processes to improve patient flow: a cross sectional study. BMC Health Serv Res 2012;12:180.

5. Chaboyer W, Wallen K, Wallis M, et al. Whiteboards: one tool to improve patient flow. Med J Aust 2009;190(11 Suppl):S137-40.

6. Sehgal NL, Green A, Vidyarthi AR, et al. Patient whiteboards as a communication tool in the hospital setting: a survey of practices and recommendations. J Hosp Med 2010;5:234-9.

7. Hessels A, Flynn L, Cimiotti JP, et al. Impact of heath information technology on the quality of patient care. Online $J$ Nurs Inform 2015;19.

8. Mathews KS, Corso P, Bacon S, et al. Using the red/yellow/green discharge tool to improve the timeliness of hospital discharges. Jt Comm J Qual Patient Saf 2014;40:243-AP3.

9. Patel H, Morduchowicz S, Mourad M. Using a systematic framework of interventions to improve early discharges. Jt Comm J Qual Patient Saf 2017;43:189-96.

10. White CM, Statile AM, White DL, et al. Using quality improvement to optimise paediatric discharge efficiency. BMJ Qual Saf 2014;23:428-36.

11. Maloney CG, Wolfe D, Gesteland PH, et al. A tool for improving patient discharge process and hospital communication practices: the "Patient Tracker". AMIA Annu Symp Proc 2007:493-7.

12. Benneyan JC, Lloyd RC, Plsek PE. Statistical process control as a tool for research and healthcare improvement. Qual Saf Health Care 2003;12:458-64.

13. Gurses AP, Xiao Y. A systematic review of the literature on multidisciplinary rounds to design information technology. J Am Med Inform Assoc 2006;13:267-76. 